\title{
A NEW TECHNIQUE OF INCOHERENT COMPLEX SIGNAL DETECTION
}

\author{
F.T.S. YU* \\ Electro-Optics Laboratory, Electrical and Computer Engineering Department, University of Michigan, \\ Ann Arbor, Michigan 48104, USA
}

Received 2 May 1978

\begin{abstract}
A new technique of incoherent complex spatial filtering is presented. The advantage of this technique is its use of white light, for which the unavoidable coherent noise in the coherent optical processor may be removed. Although the technique is effective only in one dimension, it may be applied to some problems in complex spatial filterings, for example, image enhancement, image restoration, smear correction, etc.
\end{abstract}

\section{Introduction}

One of the most interesting and important applications of optical information processing is signal detection by complex spatial filtering [1-6]. However, most of the complex optical processing techniques require a coherent source. But coherent optical processing systems are plagues with the well-known artifact noise, which in practice is unavoidable.

Although techniques of optical information processing by incoherent light are available [5-7], they generally have a serious shortcoming; namely the production of error terms due to the bias level in the signal and other transparencies.

In this letter, we propose a new technique that permits signal detection by complex spatial filtering to be carried out with a spectrally incoherent light source (that is, a white light source). Our processing system can be used for signal detection (i.e. matched filtering). We also note that this incoherent processing technique may be extended to more general complex spatial filtering problems, such as image enhancement, image restoration, etc.

\section{Incoherent complex spatial filtering}

We now discuss the application of this technique to signal detection. We place a diffraction grating behind an input-signal transparency, $s(x, y)$, at the input plane $\mathrm{P}_{1}$ of an incoherent optical processor, as shown in fig. 1. The resultant complex amplitude transmittance function of the input plane is then

$$
s(x, y) T(x)=K s(x, y)\left[1+\cos \left(p_{0} x\right)\right],
$$

where $T(x)$ represents the diffraction grating, $K$ is a proportionality constant, $p_{0}$ is the angular spatial frequency of the diffraction grating, and $(x, y)$ is the spatial coordinate system.

Since the input plane is illuminated by collimated white light, the complex light distribution at the back focal plane $\mathbf{P}_{2}$ of the transform lens, may be written

\footnotetext{
* On Sabbatical leave from the Department of Electrical and Computer Engineering, Wayne State University, Detroit, Michigan 48202, USA.
} 


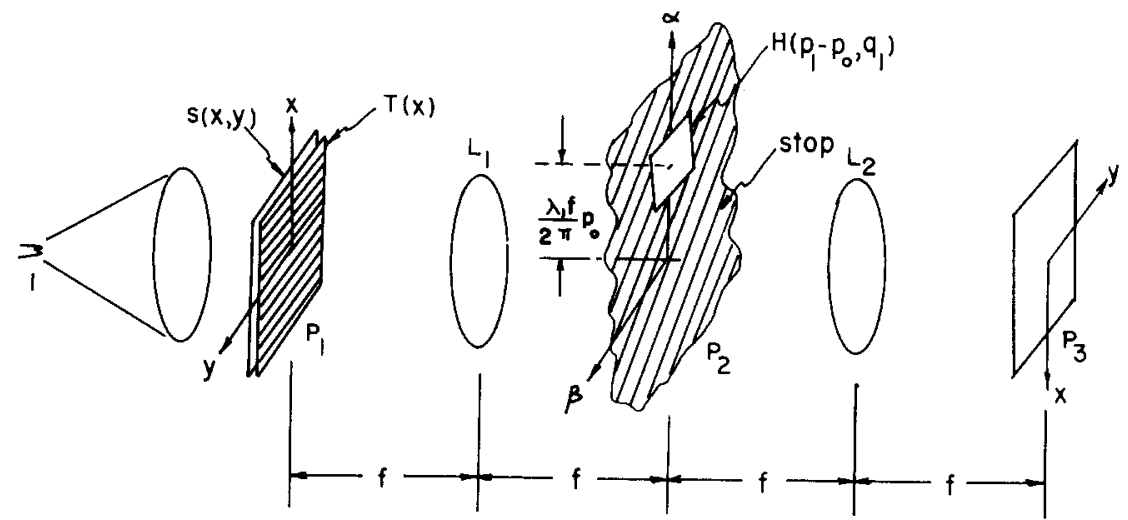

Fig. 1. An incoherent optical processor. $s(x, y)$, input signal transparency; $T(x)$, diffraction grating; $H\left(p_{1}-p_{0}, q_{1}\right)$, spatial filter, and $I$, incoherent point source.

$$
E(p, q)=C \iiint s(x, y)\left[1+\cos \left(p_{0} x\right)\right] \exp [-\mathrm{i}(p x+q y)] \mathrm{d} x \mathrm{~d} y \mathrm{~d} \lambda
$$

where the integral is over the spatial domain and spectral bandwidth $\lambda$ of the light source, $(p, q)$ denotes the angular spatial frequency coordinate system, and $C$ is a complex constant

For simplicity of analysis, we evaluate eq. (2) for a given wavelength $\lambda$, i.e.

$$
\begin{aligned}
& E(p, q, \lambda)=C \iint s(x, y)\left[1+\cos \left(p_{0} x\right)\right] \exp [-\mathrm{i}(p x+q y)] \mathrm{d} x \mathrm{~d} y \\
& \quad=C_{1} S(p ; q)+C_{2} S\left(p-p_{0}, q\right)+C_{3} S\left(p+p_{0}, q\right),
\end{aligned}
$$

where the $C$ 's are the appropriate complex constants, and

$$
S(p, q)=\iint s(x, y) \exp [-\mathrm{i}(p x+q y)] \mathrm{d} x \mathrm{~d} y,
$$

the Fourier spectrum of $s(x, y), p=(2 \pi / \lambda f) \alpha$ and $q=(2 \pi / \lambda f) \beta$ are the angular spatial frequency coordinates, $(\alpha, \beta)$ denotes the linear spatial coordinates system of $(p, q)$, and $f$ is the focal length of the transform lens.

We note that, eq. (3) can also be expressed in terms of the spatial coordinates of $\alpha$ and $\beta$, that is,

$$
E(\alpha, \beta ; \lambda)=C_{1} S(\alpha, \beta)+C_{2} S\left(\alpha-(\lambda f / 2 \pi) p_{0}, \beta\right)+C_{3} S\left(\alpha+(\lambda f / 2 \pi) p_{0}, \beta\right) .
$$

From the above equation, we note that the two first-order signal spectra (i.e. second and third terms) are dispersed into different rainbow colors [8,9] of the signal spectrum along the $\alpha$ axis, and centered at

$$
\alpha= \pm(\lambda f / 2 \pi) p_{0} .
$$

Thus we see that the origin of the differently colored signal spectra is linearly dispersed over the spatial frequency coordinate $\alpha$. We emphasize that it is the diffraction grating, at the input plane, that causes this dispersive effect. i.e.

In the analysis of complex signal detection, we assume that a complex spatial filter for $\lambda=\lambda_{1}$, is constructed,

$$
H\left(p_{1}, q_{1}\right)=K_{1}^{2}+K_{2}^{2} \mid\left(\left.S\left(p_{1}, q_{1}\right)\right|^{2}+2 K_{1} K_{2}\left|S\left(p_{1}, q_{1}\right)\right| \cos \left[\beta_{0} q_{1}+\phi\left(p_{1}, q_{1}\right)\right],\right.
$$

where $p_{1}=\left(2 \pi / \lambda_{1} f\right) \alpha, q_{1}=\left(2 \pi / \lambda_{1} f\right) \beta, \beta_{0}$ is the spatial frequency in the $q$ (or $\beta$ ) direction, $K$ 's are the appropriate constants, and 


$$
S\left(p_{1}, q_{1}\right)=\left|S\left(p_{1}, q_{1}\right)\right| \exp \left[\mathrm{i} \phi\left(p_{1}, q_{1}\right)\right],
$$

the complex signal spectrum of $s(x, y)$.

We note that the complex filter of eq. (6) can be generated by the interferometric technique of Vander Lugt [1-6]

If we insert the complex spatial filter of eq. (6) in the spatial frequency domain $\mathrm{P}_{2}$, but centered at

$$
\alpha=\left(\lambda_{1} f / 2 \pi\right) p_{0},
$$

then the complex light field (for a given $\lambda$ ) immediately behind the filter plane is

$$
E(p, q ; \lambda)=C S\left(p-p_{0}, q\right) H\left(p_{1}-p_{0}, q_{1}\right),
$$

where $H\left(p_{1}-p_{0}, q_{1}\right)$ represents the corresponding filter translation that centers at $\alpha=\left(\lambda_{1} f / 2 \pi\right) p_{0}$ and $\beta=0$.

With reference to the incoherent optical processor of fig. 1 , we see that the output complex light field at $P_{3}$ is

$$
g(x, y ; \lambda)=C \iint S\left(p-p_{0}, q\right) H\left(p_{1}-p_{0}, q_{1}\right) \exp \left[\mathrm{i}\left(p_{1} x+q_{1} y\right)\right] \mathrm{d} p_{1} \mathrm{~d} q_{1},
$$

where the integral is over the spatial domain of $H\left(p_{1}-p_{0}, q_{1}\right)$. We also assume that $H\left(p_{1}-p_{0}, q_{1}\right)$ is spatial frequency limited.

First, we evaluate eq. (10) for the case $\lambda=\lambda_{1}$. By substituting eq. (6) into eq. (10), we obtain

$$
\begin{aligned}
& g\left(x, y ; \lambda_{1}\right)=s(x, y) \exp \left(\mathrm{i} p_{0} x\right)+s(x, y) \exp \left(\mathrm{i} p_{0} x\right) * s(x, y) \exp \left(\mathrm{i} p_{0} x\right) * s^{*}(x, y) \exp \left(\mathrm{i} p_{0} x\right) \\
& \quad+s(x, y) \exp \left(\mathrm{i} p_{0} x\right) * s\left(x, y+\beta_{0}\right) \exp \left(\mathrm{i} p_{0} x\right)+s(x, y) \exp \left(\mathrm{i}_{0} x\right) * s^{*}\left(-x,-y+\beta_{0}\right) \exp \left(\mathrm{i} p_{0} x\right),
\end{aligned}
$$

where $*$ denotes the convolution operation and the superscript $*$ denotes the complex conjugate. For simplicity, the proportionality constants $K$ were dropped from evaluation.

From eq. (11) we see that the first and second terms represent the zero order terms, which are diffracted in the neighborhood of $(0,0)$ in the output plane, and the third and fourth terms are the convolution and correlation terms, which are diffracted in the neighborhood of $\left(0,-\beta_{0}\right)$ and $\left(0, \beta_{0}\right)$ respectively, as shown in fig. 2 . For the time being, the zero order and the convolution terms are assumed of no particular interest to us; it is the correlation terms that one uses for signal detection.

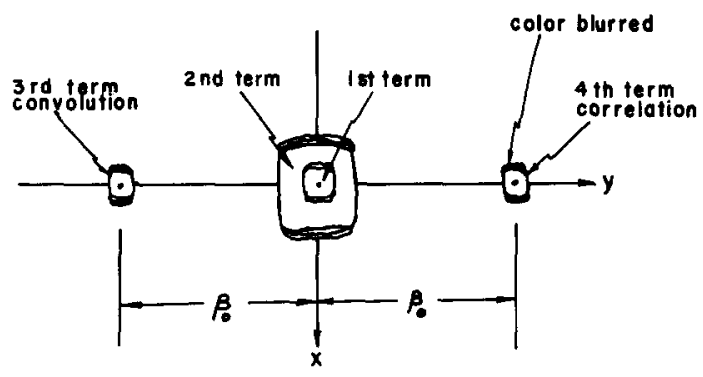

Fig. 2. Sketch of the output diffraction of a complex spatial filtering.

We now discuss the case $\lambda \neq \lambda_{1}$. Since it is our present objective to investigate complex signal detection, we therefore focus our attention on the correlation term, i.e.

$$
R(x, y ; \lambda)=\iint S\left(p-p_{0}, q\right) S^{*}\left(p_{1}-p_{0}, q_{1}\right) \exp \left\{\mathrm{i}\left[p_{1} x+\left(q_{1}-\beta_{0}\right) y\right]\right\} \mathrm{d} p_{1} \mathrm{~d} q_{1},
$$

where $p=(2 \pi / \lambda f) \alpha, q=(2 \pi / \lambda f) \beta, p_{1}=\left(2 \pi / \lambda_{1} f\right) \alpha$, and $q_{1}=\left(2 \pi / \lambda_{1} f\right) \beta$. 
Alternatively, eq. (12) can be written,

$$
R(x, y ; \lambda)=K \iint S\left(\alpha-(\lambda f / 2 \pi) p_{0}, \beta\right) S^{*}\left(\alpha-\left(\lambda_{1} f / 2 \pi\right) p_{0}, \beta\right) \exp \left(-\mathrm{i} \beta_{0} y\right) \exp \left[\mathrm{i}\left(2 \pi / \lambda_{1} f\right)(\alpha x+\beta y)\right] \mathrm{d} \alpha \mathrm{d} \beta
$$

From the above equation, we see that there is a mismatch in location of the incoming signal spectrum with respect to the filter function. Thus for two sufficiently different wavelengths, i.e.

$$
\Delta \alpha=\left(f p_{0} / 2 \pi\right)\left|\lambda-\lambda_{1}\right|,
$$

where $\Delta \alpha$ denotes the incremental separation. We see that the correlation function of eq. (13) is very weak. In other words, if the spatial carrier frequency $p_{0}$ of the diffraction grating is high, a narrower color spread of the correlation peak can be obtained. We note that the color spread exists only in the $x$ direction. That is the resolution (or accuracy) [10] of the correlation peak in the $x$ direction is expected to be somewhat lower than that in the $y$ direction. Thus, we see that this incoherent processing technique is effective only in one dimension., In other words, for some two dimensional processings, this technique may pose some drawbacks.

Finally, we emphasize that this technique will offer a lesser speckle effect in complex signal detection and it can also be extended to the application of some problems in complex spatial filterings.

\section{Conclusion}

We have introduced a new technique in incoherent complex signal filtering. Although the color spread (i.e. the accuracy) of the correlation detection is somewhat broader in $x$ direction than in the $y$ direction, the result can be significantly improved by simply increasing this spatial carrier frequency of the diffraction grating. We note that diffraction efficiency can also be improved if a thin dichromated [11] diffraction grating is used.

Since all spatial filters, including real and pure phase, can be synthesized by the interferometric technique, this new technique can therefore also be applied to some problems in the complex spatial filterings. In short, this incoherent optical processing technique may be extended to the application of image enhancement, image restoration, smear correction, etc.

Finally, the basic advantage of this technique is a white light processing capability in which the coherent noise in the processing system may be considerably reduced.

We wish to acknowledge Professor E.N. Leith for his valuable comments and suggestions during the course of this work, and we acknowledge the support of the Air Force Office of Scientific Research (Contract AFOSR773356).

\section{References}

[1] A. Vander Lugt, IEEE IT 10 (1964) 139.

[2] A. Vander Lugt, Opt. Acta 15 (1968) 1.

[3] F.T.S. Yu, IEEE IT 17 (1971) 524.

[4] F.T.S. Yu and G.C. Kung, J. Opt. Soc. Am. 16 (1972) 147.

[5] F.T.S. Yu, Introduction to diffraction information processing, and holography (MIT Press, Mass., 1973) Ch. 7.

[6] J.W. Goodman, Introduction to Fourier optics (McGraw-Hill, New York, 1968) Ch. 7.

[7] M.A. Monahan, K. Bromley and R.P. Bocker, Proc. IEEE 65 (1977) 121.

[8] E.N. Leith and B. Chang, Appl. Opt. 12 (1973) 1957.

[9] E.N. Leith and J. Roth, Appl. Opt. 16 (1977) 2565.

[10] F.T.S. Yu, Optics and information theory (Wiley-Interscience, New York, 1976) p. 127.

[11] S.K. Case and R. Alferness, Appl. Phys. 10 (1976) 41. 